Normal and shear forces in the contact patch of a braked racing tyre Part 2: Development of a physical tyre model

\author{
Patrick Gruber*, Robin S Sharp, and Andrew D Crocombe
}

Division of Mechanical, Medical, and Aerospace Engineering, Faculty of Engineering and Physical Sciences, University of Surrey, Guildford, United Kingdom

*Corresponding author: Division of Mechanical, Medical, and Aerospace Engineering (A1), Faculty of Engineering and Physical Sciences, University of Surrey, Guildford, GU2 7XH, UK. email: p.gruber@ surrey.ac.uk

This is a preprint of an article whose final and definitive form has been published in Vehicle System Dynamics ( ) 2012, copyright Taylor \& Francis; Vehicle System Dynamics is available online at: $\underline{w w w . t a n d f o n l i n e . c o m / 10.1080 / 00423114.2011 .586429 . ~}$ 


\section{Normal and shear forces in the contact patch of a braked racing tyre Part 2: Development of a physical tyre model}

This is the second part of a two-part article looking at carcass deflections, contact pressure and shear stress distributions for a steady-rolling, slipping and cambered tyre. In the first part, a previously-described and validated finite-element model of a racingcar tyre is developed further to extract detailed results which are not easily obtainable through measurements on an actual tyre. Generally, these results aid understanding of contact patch characteristics. In particular, they form a basis for the development of a simpler physical tyre model, which forms the focus of this part of the article. The created simpler tyre model has three purposes: (i) to reduce computational demand while retaining accuracy; (ii) to allow identification of tyre model features that are fundamental to an accurate representation of the contact stresses; and (iii) to create a facility for better understanding of tyre wear mechanisms and thermal effects. Results generated agree well with the physically realistic rolling-tyre behaviour demonstrated by the FE model. Also, the model results indicate that an accurate simulation of the contact stresses requires detailed understanding of carcass deformation behaviour.

Keywords: tyre model, braking, normal forces, shear forces, contact patch, racing tyre

\section{Introduction}

The contact stresses at the interface of a braked racing tyre with the ground surface have been studied in Part 1 of this article [1] with a previously-described and validated finite-element (FE) model [2]. Detailed investigation of the deflection behaviour of the rolling tyre provided novel insights into the interrelationship between the reinforcement layers (i.e., carcass) and the shear stress distribution within the contact patch. Based on these findings, this part of the article develops a simpler Physical tyre model, the purpose of which is three-fold: 1) to compute rapid solutions, which are accurate over the full operating range; 2) to establish, and allow the demonstration of those features of the tyre structure that are fundamental to the simulation of realistic normal and shear forces within the contact patch; and 3) to create a facility for the better understanding of tyre wear mechanisms and thermal effects. To achieve the three objectives, a model is required that allows individual tyre model features to be examined and their significance with respect to the generation of contact stresses throughout the entire contact patch to be studied.

The literature provides a relatively large number of physical tyre models of varying complexity which were developed to simulate the contact behaviour of a rolling tyre (e.g., [3, 
4]). One of the most well-known, simpler physical tyre models is the brush model (e.g., [5, 6]), which consists of a row of elastic bristles or tread elements attached to a rigid carcass. With this model, all deformations a tyre experiences during operation (i.e., the distortion of the carcass, the belt and the tread) are assumed to occur through the compliance of the bristles. The shear forces are generated based on a separation of the contact patch in a sliding and an adhesion region, making the brush model an instructive tool that provides basic understanding of the rolling behaviour of a tyre. Building on the concept of the brush model, Pacejka [5] developed the tread element simulation (TreadSim) model that allows investigation of more advanced tyre features such as complex rubber-to-road friction properties or consideration of camber effects. Owing to the flexibility of TreadSim to simulate the contact behaviour, its modelling approach is chosen as the basis for the physical model developed here. As will be explained below, by using data extracted from the finite element model to provide parametric information, the TreadSim approach will be extended, creating a novel physical tyre model.

The developed model is evaluated by comparing its characteristics with the behaviour of the FE tyre model.

\section{Overview of the physical model}

The physical model described here is created on the basis of the TreadSim model, which is developed around a simplified physical structure of a real tyre. TreadSim consists of three main structural tyre elements: a rigid wheel rim, a flexible carcass and a rubber tread that is discretised into an array of brush-type bristles or tread elements. The bristles are evenly distributed over a rectangular contact patch area and can only deflect in the longitudinal and lateral directions in response to the prevailing slip condition and carcass deformations. In the vertical direction, the tread elements are loaded with the normal tyre force which is assumed to follow a parabolic distribution along the longitudinal contact dimension while being 
uniform in the lateral direction. At the core of TreadSim is the tread-element-following method $[5,7,8]$, which allows simulation of advanced tyre features such as complex rubber friction properties (see section 3.3 for a detailed explanation of the method). For the results presented by Pacejka [5], a sliding-speed-dependent friction coefficient is employed, with the sliding speed approximated as the velocity of the tread belt. The flexible carcass model included in TreadSim possesses lateral, bending and yaw compliances and considers influences due to camber, conicity, ply-steer, side force and aligning moment. The specific deflection characteristics caused by these five factors are developed from theoretical concepts based on a tyre rolling on a frictionless surface. In general, the lateral deflection of the tread belt is derived from the assumption of a parabolic baseline that is modified by two parameters: first, an 'average slope' parameter which is influenced by the aligning torque and ply-steer, and, second, a 'curvature' parameter that is a function of side force, camber and conicity. To account for a finite tread width, several parallel bristle rows can be simulated and a linear variation of the rolling radius (see section 3.3.2) about the wheel centre plane is included. In addition, TreadSim contains many additional coefficients and parameters to "tweak" the model to obtain qualitatively and quantitatively sensible results.

The physical tyre model described here adopts the basic structure of TreadSim and enhances it by formulating novel mathematical expressions for the individual model elements. In contrast to TreadSim, these sub-models are derived from characteristics that are extracted from the virtual tyre (as presented in the first part of this article) rather than theoretical concepts. As a consequence, further modelling aspects are incorporated to increase simulation accuracy while additional "tweaking" coefficients are avoided. The major changes and extensions over TreadSim include a two-dimensional (2D) contact patch geometry that varies with camber angle and normal load, and considers the tread pattern. A flexible carcass is incorporated that replicates the characteristics of the tyre structure indentified in the first part of this paper, i.e., longitudinal and lateral deflections related to contact patch waisting, 
camber angle, ply-steer and horizontal tyre forces are simulated. Furthermore, the shear force computation algorithm is altered to allow non-isotropic bristle stiffness and to avoid approximate expressions used in TreadSim for the calculation of the sliding distance of a tread element.

The physical tyre model is developed to retain accuracy over the full operating range investigated with the virtual tyre which includes vertical loads of 1500, 3000 and $4500 \mathrm{~N}$, camber angles of $0^{\circ}$ and $-3^{\circ}$, and longitudinal slips from $0 \%$ to $-20 \%$.

\section{Description of the model}

Following the general structure of TreadSim, the physical tyre model comprises four sub-models that compute the following four properties: (i) carcass deflection, (ii) contact patch dimensions, (iii) contact pressure distribution and (iv) shear stress distribution. As the outputs of the model - the shear stress distribution and tyre forces - influence the deflection characteristics of the carcass, an iterative loop may be required to obtain an accurate solution (Figure 1).

Detailed descriptions of the individual model elements are presented in the following four sections.

\subsection{Contact patch geometry}

For an efficient computation of the $2 \mathrm{D}$ tread-road interface, the contact patch shape is simplified as a row of rectangular regions of contact and non-contact (Figure 2). Based on the results from the previously-described experimental investigations [2] and the FE simulations presented in the first of this pair of papers [1], the widths of the non-contacting regions (or grooves) can be assumed to stay constant over the investigated operating range. As a consequence, the inner contacting regions (ribs 2 to 4 ) are considered to have a fixed width. 
The remaining dimensions of the 2D contact patch are calculated from basic data that are extracted from FE simulations of a tyre rolling freely on a friction surface (see Part 1 for the specific analysis setup [1]). To enhance the flexibility of the model, the tread-road interface dimensions are obtained by calculation of the following four quantities: (1) contact area, (2) radial tyre deflection in the wheel centre plane, (3) contact lengths and (4) contact widths.

\subsubsection{Contact area}

FE results and corresponding published data [9] indicate that the area of the contact patch is directly dependent on the applied normal load. Also, over the investigated operating range, camber angle and slip show negligible influence on the size of the contact patch. Hence, the contact area is computed with the following linear function (Figure 3):

$$
A R E A_{\text {total }}\left(F_{z}\right)=m_{A R E A} F_{z}+\text { constant }
$$

where: $F_{z}$ : vertical tyre load

$m_{A R E A}:$ coefficient which describes the slope of the load-contact area relationship constant term: accounts for initial nonlinearity at low vertical loads [2]

\subsubsection{Radial tyre deflection}

Load-displacement tests with the freely rolling virtual tyre show that the radial tyre deflection within the centre plane of the wheel is primarily a function of normal load and camber angle. This relationship can be expressed with the following algorithm (Figure 4):

$$
\delta_{r}\left(F_{z}, \gamma\right)=\left(c_{1} F_{z}^{1 / 2}+c_{2} F_{z}\right)+\left(c_{3} F_{z}^{1 / 2}-c_{4}\right) \sin |\gamma|
$$

where: $\quad \delta_{r}$ : radial tyre deflection in the wheel centre plane

$c_{1}$ to $c_{4}$ are parameters fitted to the FE simulation results $\gamma$ : camber angle 


\subsubsection{Contact lengths}

The length of the contact patch is a geometrical property of the tyre $[5,11]$ and, hence, is calculated as a function of the radial tyre deflection (equation (2)) and the camber angle.

Extending the contact length model proposed by Besselink [5] for the observed camber angle influence, the following relationship is found (Figure 5):

$$
a_{R i b, i}\left(\delta_{r}, \gamma\right)=R_{o}\left[q_{R a 1, i}\left(\frac{\delta_{r}-(3 / 2)^{1 / 2} y_{R i b, i} \sin \gamma}{R_{o}}\right)^{1 / 2}+q_{R a 2, i} \frac{\delta_{r}-(3 / 2)^{1 / 2} y_{R i b, i} \sin \gamma}{R_{o}}\right] \text { (2) }
$$

where: $\quad a_{R i b, i}:$ half contact length of the individual tread rib

$R_{0}$ : unloaded tyre radius

$\delta_{r}$ : radial tyre deflection in the wheel centre plane

$\gamma:$ camber angle

$y_{R i b, i}:$ lateral coordinate of the centre of the individual tread rib

$q_{R \mathrm{a} 1, i}$ and $q_{R \mathrm{a} 2, i}:$ FE data fitted coefficients

\subsubsection{Contact widths}

As mentioned above, the widths of the inner tread ribs are considered to be constant. The calculation of the widths of the outermost tread ribs, in turn, requires consideration of two additional relationships. First, the combined contact area of the outermost tread ribs is determined from the difference between the total contact area (equation (1)) and the area of the inner tread ribs, i.e.:

$$
A R E A_{R i b 1+5}=A R E A_{\text {total }}-\sum_{i=2}^{4} 2 a_{R i b, i} w_{R i b, i}
$$

Second, the longitudinal and lateral contact dimensions of the outermost ribs are related to each other by their relative ratios (Figure 6):

$$
\frac{a_{R i b 1}}{a_{R i b 5}}=\frac{w_{R i b 1}}{w_{R i b 5}}
$$

where: $\quad a_{R i b 1}$ and $a_{R i b 5}$ : half contact lengths of outermost tread ribs $w_{R i b 1}$ and $w_{R i b 5}$ : contact widths of outermost tread ribs 
Then, using equation (5) together with equations (3) and (4), the contact widths of the outermost tread ribs become:

$$
w_{R i b 1}=\frac{\operatorname{AREA}_{\text {total }}-\sum_{i=2}^{4} 2 a_{R i b, i} w_{R i b, i}}{2\left(a_{R i b 1}+\frac{a_{R i b 5}^{2}}{a_{R i b 1}}\right)} \text { and } \quad w_{R i b 5}=w_{R i b 1} \frac{a_{R i b 5}}{a_{R i b 1}}
$$

\subsection{Contact pressure}

In accordance with common modelling techniques, the contact pressure distribution is assumed to be uniform across the width of the individual tread ribs and allowed to vary in the longitudinal direction (Figure 7). Also, similar to the TreadSim approach, slip and direct camber angle influences are disregarded here (camber effects are indirectly included via the change in contact length (equation (2)) and the normal load share per tread rib (equation (9)). For the model development, data on the normal force distribution extracted from FE simulations of the tyre rolling freely on a friction surface at different vertical loads are analysed.

To allow close replication of the normal pressure distribution predicted by the virtual tyre, a contact pressure algorithm based on the model proposed by Guo and $\mathrm{Lu} \mathrm{[12]} \mathrm{is}$ employed:

$$
p_{z, i}\left(x_{R i b, i}\right)=\frac{F_{z, i}}{2 a_{R i b, i} w_{R i b, i}} \eta\left(\frac{x_{R i b, i}}{a_{R i b, i}}\right)
$$

where: $\quad F_{z, i}:$ normal tyre load of tread rib $i$

$x_{R i b, i}$ longitudinal coordinate of tread rib $i$ with respect to the contact patch centre

$a_{R i b, i}:$ half contact length of tread rib $i$

$w_{R i b, i}:$ contact width of tread rib $i$

$$
\eta\left(\frac{x_{R i b, i}}{a_{R i b, i}}\right) \text { is given as: } \eta\left(\frac{x_{R i b, i}}{a_{R i b, i}}\right)=c_{R i b, i}\left[1-\left(\frac{x_{R i b, i}}{a_{R i b, i}}\right)^{2 n_{R i b, i}}\right]\left[1+\lambda_{R i b, i}\left(\frac{x_{R i b, i}}{a_{R i b, i}}\right)^{2 n_{R i b, i}}\right]
$$


with: $\quad c_{R i b, i}=\frac{\left(2 n_{R i b, i}+1\right)\left(4 n_{R i b, i}+1\right)}{2 n_{R i b, i}\left(4 n_{R i b, i}+1+\lambda_{R i b, i}\right)}$

$n_{R i b, i}, \lambda_{R i b i}$ : fitted coefficients which are functions of vertical load

Owing to an almost even distribution of the vertical tyre load over the entire contact patch, the normal load of the individual tread rib $\left(F_{z, i}\right)$ can be obtained from a direct relationship between the normal load share and the contact area share of each tread rib (Figure 8), i.e.:

$$
\frac{F_{z R i b, i}}{F_{z}}=\frac{A R E A_{\text {Rib,i }}}{A R E A_{\text {total }}}
$$

\subsection{Contact shear forces}

The tyre tread is approximated as an array of elastic bristles that are arranged in parallel rows (with a minimum of one row per tread rib), which run from the leading edge to the trailing edge of the contact patch (Figure 9). The tread elements are modelled as linear springs which are attached to an inextensible belt at one end (bristle base) and contact the road surface at the other end (bristle tip). Depending on the operating condition of the tyre, frictional shear forces are generated at the bristle tips, which cause the tread elements to deflect. As only steady-state rolling conditions are investigated, the deflection of a bristle at a certain longitudinal coordinate does not change with time. Accordingly, the motion of only one bristle per row has to be monitored as it travels from the leading edge to the trailing edge of the contact patch ("tread-element-following method"). Then, with knowledge of the bristle's motion history, the deflection of all tread elements along the contact length is available and the contact forces throughout the tyre-road interface can be calculated.

Due to the strong interdependence between the carcass deformation characteristics and the shear stress distribution within the contact patch [1], the accurate computation of the deflection of the tread elements requires consideration of tyre structural effects. In particular, local rolling radius influences (see section 3.3.2) and effects arising from camber, ply-steer, 
normal load and horizontal tyre forces (see section 3.4) are included in the model.

Correspondingly, FE data obtained from rolling simulations on friction and frictionless surfaces at varying normal loads, camber angles and longitudinal slips are employed for the development of the physical model.

\subsubsection{Shear force calculation algorithm}

As mentioned above, the shear force distribution at the tread-road interface is computed from the deformation state of all bristles within the contact patch. The bristle deflection, in turn, is dependent on the prevailing rolling condition and the longitudinal and lateral tyre carcass deformations. The influence of the carcass is considered by varying the position of the belt (i.e., bristle base) as explained later, and the rolling condition is accounted for by altering the velocity of the belt. To determine the rolling condition, three model input quantities - namely, camber angle, wheel velocity (set to be equal to the velocity of the contact patch centre, i.e., the origin of the ISO-axis system) and slip (longitudinal and lateral) - are used in combination with the calculated contact patch geometry (section 3.1) and contact pressure distribution (section 3.2).

The method of monitoring one tread element per row is implemented by performing computations at fixed time intervals during which the direction of the bristle motion is considered to be constant. Assuming a steady angular velocity of the wheel $(\Omega)$ and, thus, a constant linear speed of rolling $\left(V_{r}=r_{e} \Omega\right)$ along the contact length (Figure 10), the bristle as a whole moves in one time step, $\Delta t$, over the interval $\Delta x$ towards the rear (with respect to the contact patch centre). Hence, by specifying the interval $\Delta x$ (for example, as the longitudinal distance between bristles) the time step $\Delta t$ is given by:

$$
\Delta t=\frac{\Delta x}{V_{r}}
$$


When the tyre is rolling and shear forces are generated between the tread and the road (for instance, caused by slip, camber and/or carcass deformations), the base point of the bristle (point B in Figure 10) will travel with the velocity $\boldsymbol{V}_{\boldsymbol{b}}$ relative to the ground. In the $\mathrm{x}$-direction, the speed of the bristle base is found from the longitudinal slip velocity of the tyre $\left(V_{s x}\right)$ which, for the case of a cambered tyre, is adjusted for local longitudinal speed influences, namely, rolling radius effects as explained in section 3.3.2:

$$
V_{b x}=V_{s x}+V_{s x, \gamma}
$$

where: $\quad V_{s x}=-V_{c x} \kappa:$ longitudinal slip velocity

with $V_{c x}$ : longitudinal velocity of the contact patch centre

$\kappa$ : slip ratio

$V_{s x, \gamma}$ : camber angle induced longitudinal slip velocity (equation (25))

Correspondingly, the velocity of the bristle base in the y-direction is given by the lateral tyre slip velocity that is adjusted for local lateral velocity influences induced by the lateral deformation of the carcass (see section 3.4):

$$
V_{b y}=V_{s y}-\frac{\partial y_{b}}{\partial x_{b}} V_{r}
$$

where: $\quad V_{s y}=V_{c x} \tan \alpha$ : lateral slip velocity; with $\alpha$ : slip angle

$V_{x}=V_{c x}-V_{s x}$ : longitudinal velocity of the tyre

$\partial y_{b} / \partial x_{b}$ : gradient of the carcass deflection (equation (30)) at $x_{b}=x+0.5 \Delta x$ (midpoint of the interval $\left.\Delta x\right)$

$V_{r}=r_{e} \Omega=V_{c x}-V_{s x}$ : linear velocity of rolling

Knowing the velocity vector $V_{b}$, the displacement of the base point B during one time step becomes:

$$
\Delta s_{i}=V_{b} \Delta t
$$

The deflection of the tread element, in turn, is calculated from the displacement vector $\Delta s_{i}$ and under consideration of one of two possible bristle tip (point P in Figure 10) states: (a) when the bristle tip adheres to the ground, and (b) when the bristle tip slides over the ground. 
For the case of adhesion (Figure $11 \mathrm{a}$ ), the deflection vector of the bristle after one time step $\left(\boldsymbol{e}_{i}\right)$ is given by the vector sum of the displacement vector (equation (13)) and the deflection vector of the bristle at the start of the time step $\left(\boldsymbol{e}_{i-1}\right)$ :

$$
e_{i}=e_{i-1}-\Delta s_{i}
$$

The corresponding deformation force vector of the bristle $\left(f_{i}\right)$ is obtained by the product of the bristle deformation vector and the bristle stiffness matrix $\left(\mathbf{C}_{\mathbf{i}}\right)$ :

$$
\boldsymbol{f}_{i}=\mathbf{C}_{\mathbf{i}} \boldsymbol{e}_{i}=\left[\begin{array}{cc}
c_{i x} & 0 \\
0 & c_{i y}
\end{array}\right]\left[\begin{array}{l}
e_{i x} \\
e_{i y}
\end{array}\right]
$$

The adhesion condition is maintained as long as the deformation force is smaller than the available local friction force at the bristle tip:

$$
\left|f_{i}\right|<\mu p_{z}(\Delta x \Delta y)
$$

where: $\mu$ : local friction coefficient

$p_{z}$ : local contact pressure

$\Delta x \Delta y$ : local contact area of bristle (Figure 9)

The local friction coefficient at the bristle tip is calculated at each time step with the same pressure- and sliding-speed-dependent friction model used in the FE model [1]:

$$
\mu\left(p_{z}, V_{g}\right)=\mu\left(p_{z}\right) \mu\left(V_{g}\right)
$$

As in TreadSim, the sliding-velocity of the bristle tip is approximated as the velocity of the bristle base (equation (11) and equation (12)).

When the deformation force exceeds the friction limit, the bristle starts to slide in the opposite direction to the deformation force vector (Figure $11 \mathrm{~b}$ ). Then, at the end of the time step the deflection vector of the sliding bristle is given by:

$$
\boldsymbol{e}_{i}=\boldsymbol{e}_{a d}-c_{s l} \frac{\boldsymbol{f}_{a i}}{\left|\boldsymbol{f}_{a i}\right|}
$$

where: $\boldsymbol{e}_{a d}$ : theoretical deflection vector of the bristle if the tip would adhere to the ground

$f_{a i}=\mathbf{C}_{\mathrm{i}} \boldsymbol{e}_{a d}$

$c_{s l}:$ sliding distance 
As the bristle can only be stretched to the extent that it loses grip, the sliding distance of the tread element $\left(c_{s l}\right)$ is found from the condition when the deformation force is equal to the available friction force, i.e.:

$$
\left|\mathbf{C}_{\mathbf{i}} \boldsymbol{e}_{i}\right|=\mu p_{z}(\Delta x \Delta y)
$$

Combining equations (18) and (19), and expanding the vector terms, the sliding distance is found by solving a quadratic equation:

$$
\left(\frac{e_{a d x}^{2} c_{i x}^{4}+e_{a d y}^{2} c_{i y}^{4}}{\left|f_{a i}\right|^{2}}\right) c_{s l}^{2}-\left(2 \frac{e_{a d x}^{2} c_{i x}^{3}+e_{a d y}^{2} c_{i y}^{3}}{\left|f_{a i}\right|}\right) c_{s l}+\left(e_{a d x}^{2} c_{i x}^{2}+e_{a d y}^{2} c_{i y}^{2}-\mu^{2}[p(\Delta x \Delta y)]^{2}\right)=0
$$

Knowing the sliding distance, the deflection vector and the deformation force vector of the tread element can be determined (equation (15)). To obtain the shear force distribution within the entire contact patch, the calculation steps are repeated for each time step and for each row to obtain the shear force distribution within the contact patch. Accordingly, the shear stress distribution is obtained by dividing the local bristle deformation forces by the corresponding local contact area of the bristles. Also, the total lateral and longitudinal forces generated by the tyre are given by the summation of the contact shear forces in the $\mathrm{x}$ - and $\mathrm{y}$ directions.

\subsubsection{Rolling radius}

As outlined in the first part of this two-part paper, the rolling radius of the tyre as a whole is defined as the ratio of its longitudinal velocity and its angular speed when no longitudinal force is generated. Owing to the dependence on the deformation of the tyre structure, the rolling radius varies with normal load and camber angle. Based on the FE simulation results and equivalent published data (e.g., [5]), the rolling radius reduces with increasing normal tyre load. For the investigated operating range, this normal load effect is modelled as a linear function: 


$$
r_{e}\left(F_{z}\right)=m_{r e} F_{z}+\text { constant }
$$

where: $F_{z}$ : vertical tyre load

$m_{r e}$ : fitted coefficient which describes the slope of the rolling radius against normal load relationship

The influence of camber angle is considered by varying the rolling radius across the width of the tyre. That is, the concept of a local rolling radius, as presented in Part 1 , is introduced in the model. Based on results from rolling simulations on a frictionless surface, this lateral variation due to camber angle can be approximated as a linear function of the lateral coordinate relative to the centroid of the contact patch. Hence, in combination with the overall rolling radius (equation (21)), the local rolling radius along the tyre cross-section becomes:

$$
r_{e j}\left(y_{b, c e n t r o i d}\right)=r_{e}+\Delta r_{e j} y_{b, c e n t r o i d} \sin \gamma
$$

where: $r_{e}$ : rolling radius of the tyre

$\Delta r_{e j}$ : fitted coefficient which describes the slope of the variation of the rolling radius across the tyre width

$\gamma$ : camber angle

$y_{b, \text { centroid }}$ : lateral coordinate of the undeformed bristle row with respect to the centroid of the contact patch

The lateral centroid coordinate is found from the ratio of the area of the individual contact zones weighted by their average lateral coordinate and the total contact patch area:

$$
y_{\text {centroid }}=\frac{\sum 2 a_{\text {Rib }, i} w_{R i b, i} y_{\text {Rib }, i}}{A R E A_{\text {total }}}
$$

where: $a_{R i b, i}$ : half contact length of tread rib $i$ (equation (3))

$w_{R i b, i}$ : contact width of tread rib $i$ (equation (6))

$y_{R i b, i}$ : lateral coordinate of centre of individual tread rib

With the help of the local rolling radius (equation (22)), the local longitudinal slip velocity induced by the camber angle (equation (11)) can be computed:

$$
V_{s x, \gamma}=V_{c x}-r_{e j} \Omega
$$

where: $V_{c x}$ : longitudinal velocity of the contact patch centre

$\Omega$ : angular velocity of the wheel 
Considering that the velocity of the contact patch centre is given by $V_{c x}=r_{e} \Omega$, equation (24) simplifies to:

$$
V_{s x, \gamma}=-\Delta r_{e j} y_{b, c e n t r o i d} \Omega \sin \gamma
$$

\subsection{Carcass deflection}

The influence of the compliance of the tyre structure on the contact stresses is replicated by varying the $\mathrm{x}$ - and $\mathrm{y}$-coordinates of the base points of the tread elements, which, in turn determine the gradient $\partial y_{b} / \partial x_{b}$ of the bristle rows and, thus, the lateral velocity of the bristle bases (equation (12)). Consequently, the bristle deformation characteristics and, hence, the shear forces generated at the bristle tips are altered. For the physical model, the carcass deflection characteristics identified in Part 1 are divided into two categories: (1) zero-slip influences induced by vertical load, camber angle and ply-steer, and (2) slip influences arising from longitudinal and lateral tyre forces. Both categories are described in more detail in the following two sections.

\subsubsection{Zero-slip influences}

The carcass deformations of a freely-rolling tyre are determined by the two operating conditions, normal load and camber angle, and by the tyre structure-dependent factor, ply-steer. As described in the Part 1, vertical loading of the tyre leads to lateral shrinkage in the contact region and camber angle produces lateral bending of the belt. Ply-steer causes a slight rotation of the entire belt about the vertical axis, whereby the magnitude of the rotational displacement increases with rising normal load and vice versa.

All of these influences are treated separately so that the overall belt deflection is obtained by superimposing the three effects. Based on simulation data (Part 1 [1], Figs. 8 b and $10 \mathrm{~b}$ ), the lateral deflection due to either camber angle or contact patch waisting can be approximated using a parabolic function that varies across the width of the tyre (first 
coefficients of equation (26)). The effect of ply-steer, i.e., the rotation of each bristle row, is then introduced by specifying an average slope for the parabolic base line which is dependent on the normal load (second coefficient of equation (26)). Hence, the lateral coordinate of each bristle row of a freely-rolling tyre is given by:

$$
y_{b 0}\left(x_{b}\right)=\left(c_{F z, b}+c_{\gamma, b}\right) x_{b}^{2}+r_{F z, b} x_{b}+b_{r o w}
$$

where: $\quad x_{b}=x+0.5 \Delta x:$ midpoint of the interval $\Delta x$

$b_{\text {row }}$ : lateral coordinate of the undeflected bristle row

$c_{F z, b}:$ 'curvature' parameter of the parabolic function due to normal load

$c_{\gamma, b}$ : 'curvature' parameter of the parabolic function due to camber angle

$r_{F, b}:$ 'rotation' parameter of the parabolic baseline due to ply-steer

\subsubsection{Slip influences}

On top of the deformations experienced by the tyre at zero slip, horizontal forces generated within the contact patch of the slipping tyre distort the tread belt. As explained in the FE analysis of the braked tyre (Part 1 [1], Figs. 9 b and 13 b), the influence of longitudinal and lateral forces on the tyre structure can be interpreted as a translational displacement and a rotational displacement of the belt rows. These two belt deflection mechanisms are modelled separately in the physical model.

During braking, the translational displacement of the contact patch is primarily longitudinal (towards the rear), because of the generated braking force. Also, a small lateral shift can be observed which results from the side force generated by ply-steer. Within the physical tyre model, the response to the horizontal tyre forces is simulated by allowing the bristle rows to move together in the $\mathrm{x}$ - and $\mathrm{y}$-directions with respect to the wheel centre. Specifically, virtual test data (force against displacement tests of a stationary tyre) suggest that the longitudinal and lateral carcass characteristics can be approximated as linear springs which possess stiffness constants that are dependent on the normal load:

$$
K_{x}\left(F_{z}\right)=q_{K x 1} F_{z}+q_{K x 2}
$$




$$
K_{y}\left(F_{z}\right)=q_{K y 1} F_{z}+q_{K y 2}
$$

where: $F_{z}$ : normal load

$q_{K x, y}$-terms: fitting parameters

Accordingly, the longitudinal and lateral tread displacements, which are added to the displacements of the bristle bases, are:

$$
\delta_{x}=\frac{F_{x}}{K_{x}} \quad \text { and } \quad \delta_{y}=\frac{F_{y}}{K_{y}}
$$

where: $F_{x}$ : longitudinal tyre force

$F_{y}$ : lateral tyre force

In addition to the translational displacement of the contact patch during braking, the belt contracts laterally in the leading half and expands laterally in the trailing half of the contact patch, i.e., the individual belt rows undergo a rotational motion. This behaviour, which results from a combined effect of contact patch waisting and contact patch stretching, is simulated by introducing additional terms (rotation parameters) for the average slope for the parabolic baseline of the bristle rows (equation (26)). As the rotation of the bristle rows varies across the width of the tyre, the rotation parameters are functions of the lateral coordinate:

$$
y_{b}\left(x_{b}\right)=y_{b 0}+\left(r_{F x, b}+r_{F y, b}\right) x_{b}+\delta_{y}
$$

where: $\quad y_{b 0}$ : lateral belt deflection caused by contact patch waisting and camber angle (equation (26))

$r_{F x, b}$ : 'rotation' parameter of the parabolic baseline due to $F_{x}$

$r_{F y, b}$ : 'rotation' parameter of the parabolic baseline due to $F_{y}$

$\delta_{y}$ : lateral deflection due to carcass compliance (equation (29))

\section{Verification of the model}

The developed physical model is evaluated by comparing its characteristics with the behaviour of the FE tyre model. In particular, results of the longitudinal force against slip ratio characteristics and the shear stress distributions in the contact region are presented and discussed below. 


\subsection{Slip characteristics}

As indicated by Figure 12, the longitudinal force against slip ratio curves obtained from the developed physical model exhibit the expected shape and their characteristics are similar to the FE results discussed in the first part of this article.

Quantitatively, however, notable differences in the calculated longitudinal forces at low slip ratios (between $0 \%$ and about $-12 \%$ ) exist between the different simulation methods. The physical model shows higher slip stiffnesses and greater peak force values than the FE analyses. This discrepancy can be attributed to the friction model of the finite element treatment. As described in Part 1, to avoid convergence problems the FE package approximates the condition of stick by permitting small relative sliding between the contacting surfaces before the frictional shear stress limit is reached. These numerical limitations were found to have some bearing on the computed slip stiffness of the tyre, i.e., a greater relaxation of the stick condition leads to lower tyre slip stiffnesses and vice versa. Consequently, as the developed tyre model does not require this relaxation of the stick condition, the computed slip stiffness is greater than with the FE model. In other words, if the finite element tyre model could be successfully run with a tighter friction setting, the slip characteristics of the virtual tyre would match the results of the physical model more closely. This trend is indicated by Figure 13, which plots the slip tolerance setting against the computed slip stiffness for the finite element model and the physical model developed here.

\subsection{Shear stress distribution}

The shear stress vector plots obtained with the two models (Figure 14 to Figure 17), first of all, demonstrate that the simplified contact patch dimensions of the physical model agree well with the FE simulations for the upright tyre and the cambered tyre. Also, the distributions of the shear stresses within the contact region show substantial agreement. These general observations indicate that the shear force calculation algorithm in combination with the 
flexible carcass model and the contact pressure model allows computation of realistic tread-road interaction.

Specifically, the shear stress distributions of the upright, freely-rolling tyre (Figure 14) reveal that the tyre structural effects corresponding to contact patch waisting and ply-steer are captured well with the flexible carcass model (equation (26)). Namely, lateral shrinkage of the contact patch is indicated by the shear stress vectors pointing away from the wheel centre plane and ply-steer effects are reflected by the asymmetric lateral shear stress distribution with respect to the wheel centre plane. Discrepancies between the shear stress distributions predicted by the two tyre models result primarily from three approximations employed in the developed physical model. Firstly, the assumption of a uniform contact pressure in the lateral direction does not allow a reduction of the shear stresses close to the sidewalls in the outermost tread ribs as observed with the virtual tyre. Secondly, the flexible carcass model is created to capture the major deflection characteristics of the tyre structure so that local belt deformations which induce the 'whirl' of vectors near the trailing edge are not replicated. Thirdly, local rolling radius effects are only simulated with inclined tyre configurations and, hence, the small localised longitudinal shear stresses within the individual tread ribs are not predicted.

At a slip ratio of $-10 \%$ the shear stresses produced at the tread-road interface of the upright tyre are primarily longitudinal (Figure 15), because of the dominance of the generated brake force over the side forces induced by the tyre structure. Nonetheless, the slight inclination of the shear stress vectors (primarily in the outermost tread rib) with respect to the $\mathrm{X}$-axis predicted by both models shows that the flexible carcass model captures the tyre deflection behaviour well (equation (30)). Again, the small, localised differences in the computed shear stresses can be attributed to the three modelling assumptions outlined above.

The good agreement of the contact stresses calculated by the two models for the cambered tyre at zero slip (Figure 16) implies that the superposition of the structural 
influences associated with camber angle, contact patch waisting, ply-steer and local rolling radius works well in the developed model. In particular, the 'rotating' shear stress field within the laden tread rib highlights the fact that the developed flexible carcass model is able to replicate true tyre deformation characteristics. Also, the small driving forces within the unladen side and the braking forces within the laden side of the contact patch indicate that the approximation of the linear variation of the rolling radius across the tread width (equation (22)) is sufficient to capture the general behaviour. The discrepancies between the two shear stress plots arise from modelling approximations related to contact pressure and local belt deformation characteristics.

Similar to the upright tyre, the introduction of brake slip with the cambered tyre generates shear stress vectors that are primarily pointing towards the rear. For this operating condition, the shear stresses predicted by the developed physical model match well with the corresponding finite element solution (Figure 17). This good agreement emphasises once more the fact that the superposition of the individual structural deflection mechanisms yields realistic tyre behaviour. The small differences in local shear stress patterns, in turn, can be attributed to the aforementioned modelling approximations.

\section{Conclusions}

The development of a simple physical tyre model to simulate contact patch characteristics from virtual test data has been reported. The model successfully builds on the structure of TreadSim [5] and extends it to allow simulation of tyre behaviour identified in Part 1 of this article. The major enhancements over TreadSim include: a) avoidance of non-physicallybased "model tweaking parameters"; b) a two-dimensional contact patch geometry that considers the tread pattern and varies with camber angle and normal load; c) a flexible carcass that considers deflections related to contact patch waisting, camber angle, ply-steer and horizontal tyre forces; d) a shear force computation algorithm that allows non-isotropic 
tread stiffness and avoids approximate expressions; e) lateral variation of local rolling radius about the centroid of the contact patch.

Model results show substantial agreement with virtual test results in terms of slip characteristics and shear stress distribution within the contact patch of the braked tyre. This good agreement implies that the model is capable of reproducing physically realistic behaviour of a rolling tyre. Also, the results indicate that an accurate simulation of the contact stresses requires detailed understanding of carcass deformation behaviour.

Although the outlined tyre model was developed for a grooved racing tyre, it should be noted that the presented findings are also useful for heavy wet racing tyres due to their deep tread pattern. In addition, the characteristics of tread-less racing tyres, i.e., slick tyres, can be easily incorporated in the model by reducing the gap between the contact ribs to zero or, in other words, expanding the widths of the tread ribs (equation (6)). Hence, the created physical model is applicable to a wide range of tyre types.

Current work is concerned with identification of fundamental tyre simulation parameters with the created physical tyre model.

\section{References}

[1] Gruber, P., Sharp, R. S. and Crocombe, A. D., Normal and shear forces in the contact patch of a braked racing tyre; Part 1: Results from a Finite Element model, Vehicle Syst. Dyn. 50(2) (2012), pp. 323-337.

[2] Gruber, P., Sharp, R. S. and Crocombe, A. D., Friction and camber influences on the static stiffness properties of a racing tyre, Proc. IMechE 222, Part D: J. Automobile Engineering (2008), pp. 1965-1976.

[3] Gong, S., A study of in-plane dynamics of tires, Ph.D diss., TU Delft, 1993.

[4] Kim, S., Kondo, K., Akasaka, T., Contact Pressure Distribution of Radial Tire in Motion With Camber Angle, Tire Sci. Technol. 28(1) (2000), pp. 2-32.

[5] Pacejka, H. B., Tyre and vehicle dynamics, 2nd Edition, Butterworth-Heinemann, Oxford, 2006.

[6] Gäfvert, M., Svendenius, J., A novel semi-empirical tyre model for combined slips, Vehicle Syst. Dyn.. 43(5) (2005), pp. 351-384.

[7] Pacejka, H. B., A hybrid computer model of tire shear force generation, Highway Safety Research Institute, Ann Arbor, Mich., 1971.

[8] Fancher, P., Segel, L., MacAdam, C., Pacejka, H., Tire traction grading procedures as derived from the maneuvering characteristics of a tire-vehicle system, Volume 2, Highway Safety Research Institute, Ann Arbor, Mich. 1972. 
[9] Kageyama, I., Kuwahara, S., A study on tire modeling for camber thrust and camber torque, JSAE Review 23 (2002), pp. 325-331.

[10] Zegelaar, P.W.A., The dynamic response of tyres to brake torque variations and road unevennesses, PhD. Diss., Delft University, 1998.

[11] Besselink, I., Shimmy of Aircraft Main Landing Gears, PhD. Diss., Delft University, 2000.

[12] Guo, K. and Lu, D., UniTire: unified tire model for vehicle dynamic simulation, Vehicle Syst. Dyn. Suppl. 45 (2007), pp. 79-99. 


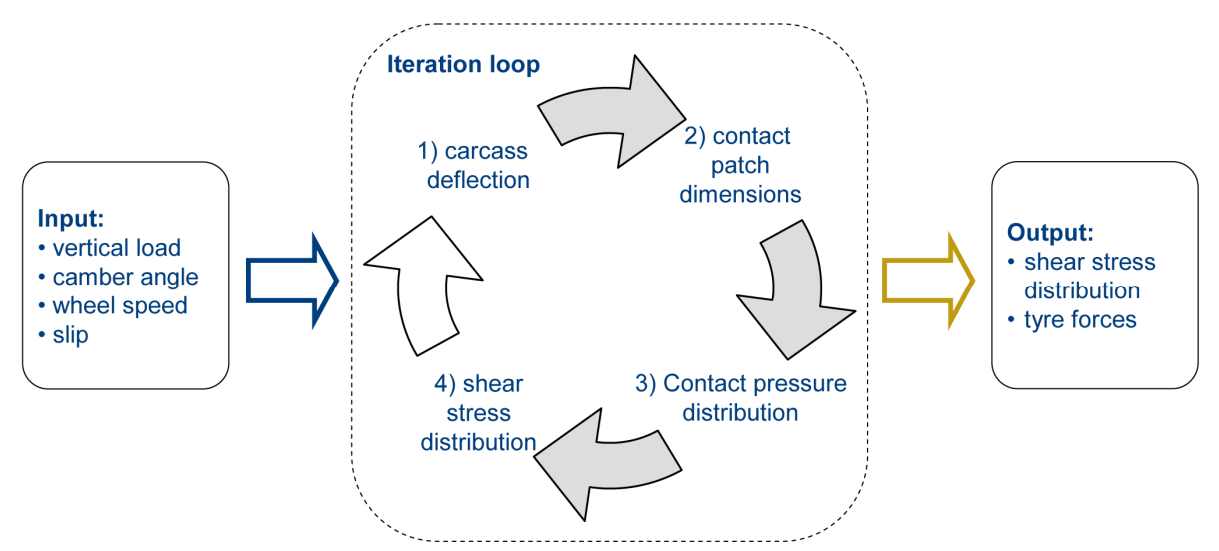

Figure 1: Structure of the physical tyre model outlining the four process stages.

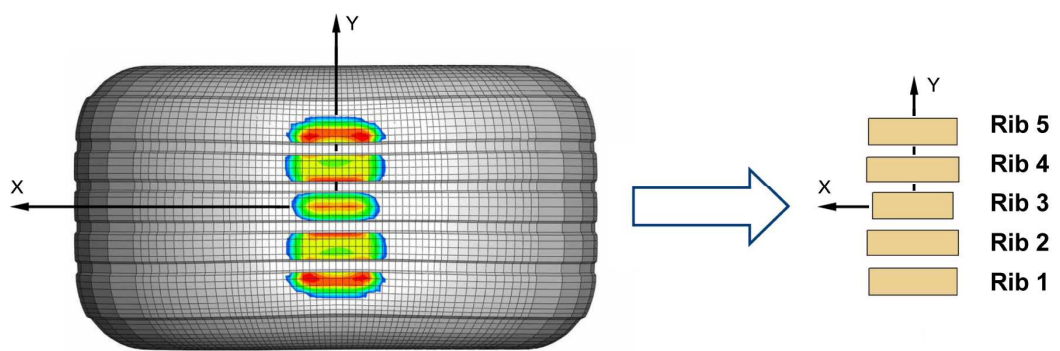

Figure 2: Approximation of the contact patch shape; left: FE model showing the normal pressure distribution within the contact patch of a freely rolling tyre; right: simplified contact patch. Both models shown from underneath.

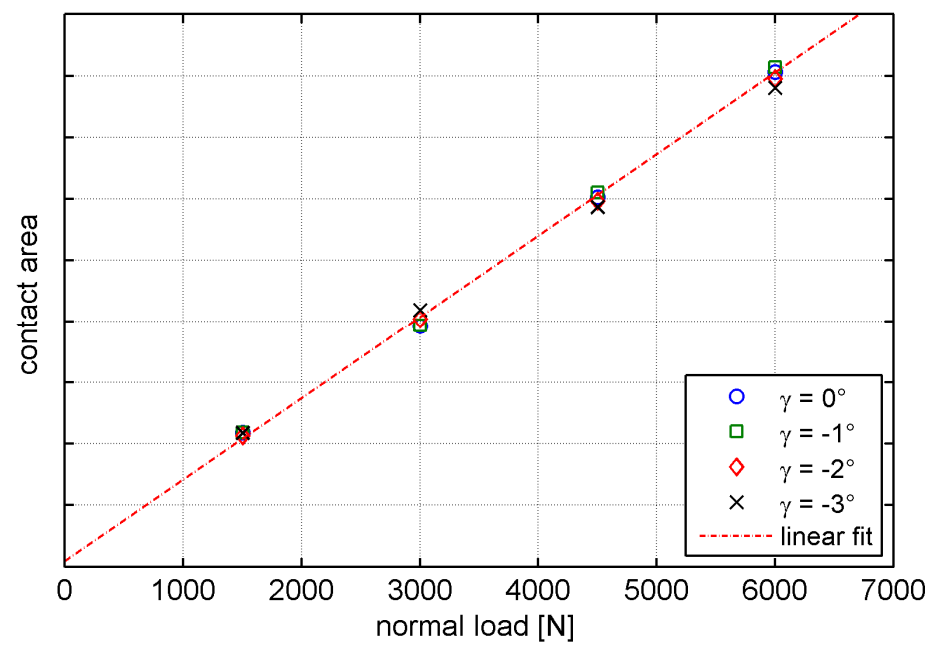

Figure 3: Contact area of the rectangular contact patch against vertical load at four different camber angles. Markers indicate FE simulation results and dashed line denotes the fitted results using equation (1). 


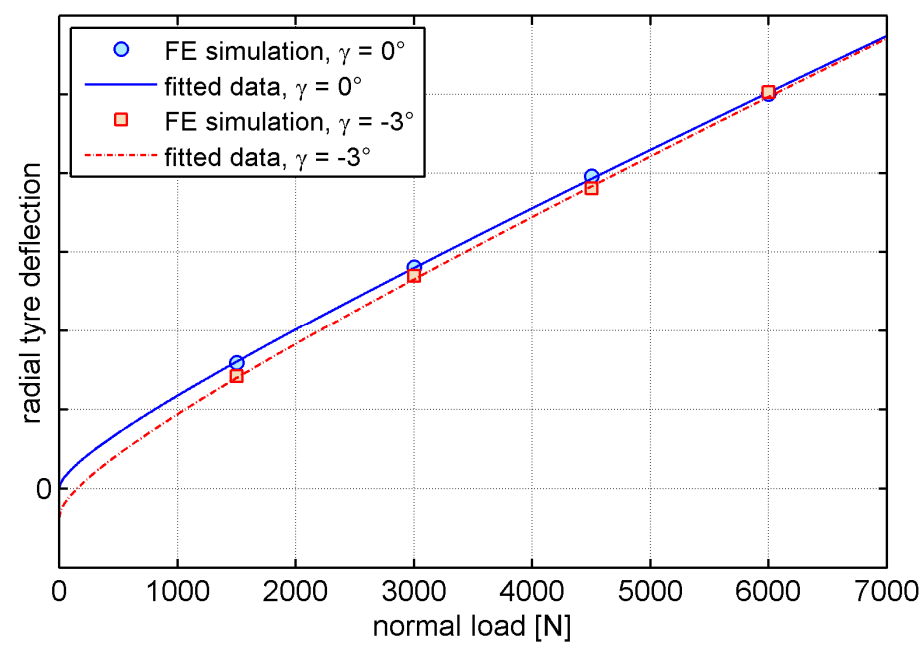

Figure 4: Radial tyre deflection against vertical load at $0^{\circ}$ and $-3^{\circ}$ camber angles. Markers denote FE simulation results and dashed lines indicate the fitted results using equation (2).

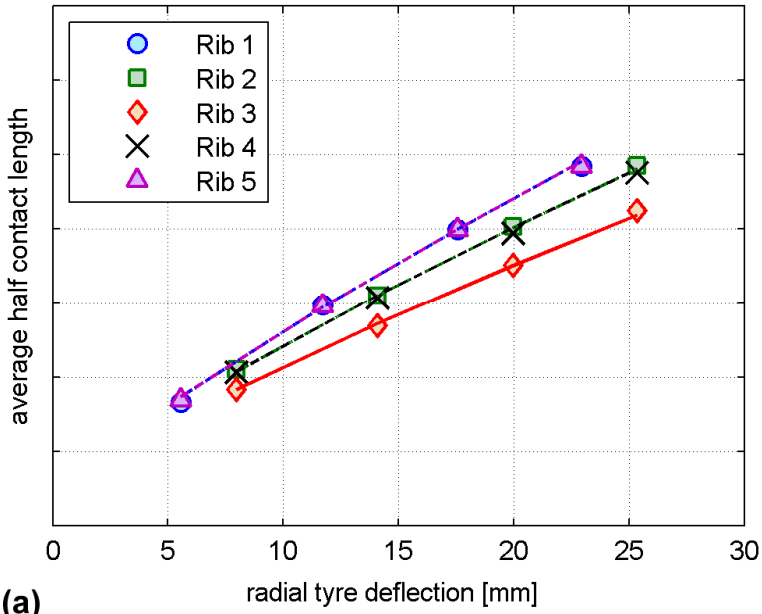

(a)

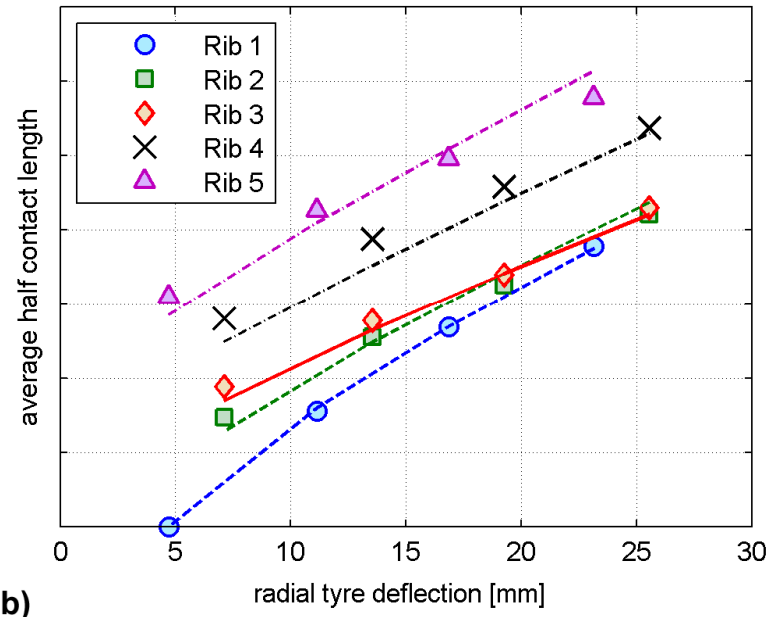

Figure 5: Simulated (markers) and fitted (lines) half contact lengths of the individual tread ribs against radial tyre deflection: (a) tyre at zero camber and (b) tyre at $-3^{\circ}$ camber. 


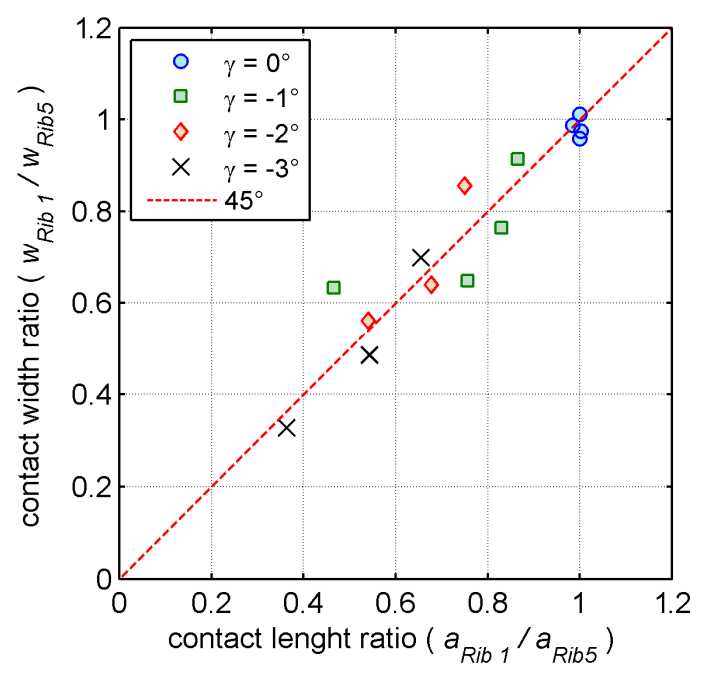

Figure 6: Contact length ratio against contact width ratio of the outermost tread ribs as functions of normal load and camber angle.

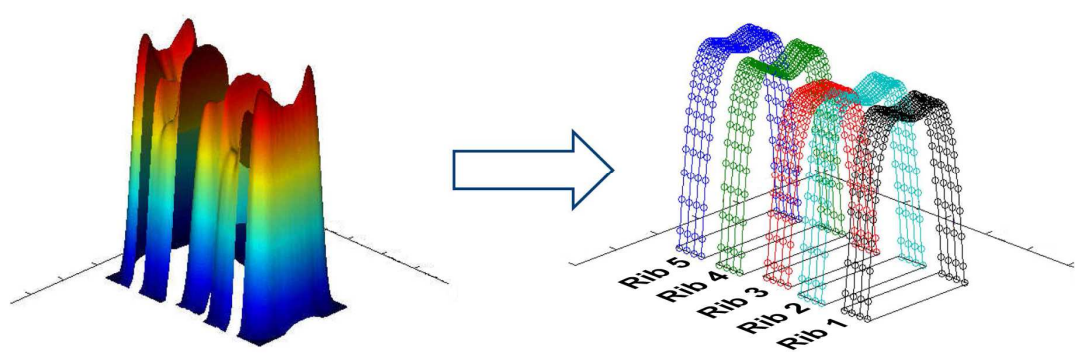

Figure 7: Modelling of the contact pressure distribution; left: contact pressure distribution extracted from FE model; right: approximated contact pressure distribution employed in the physical model.
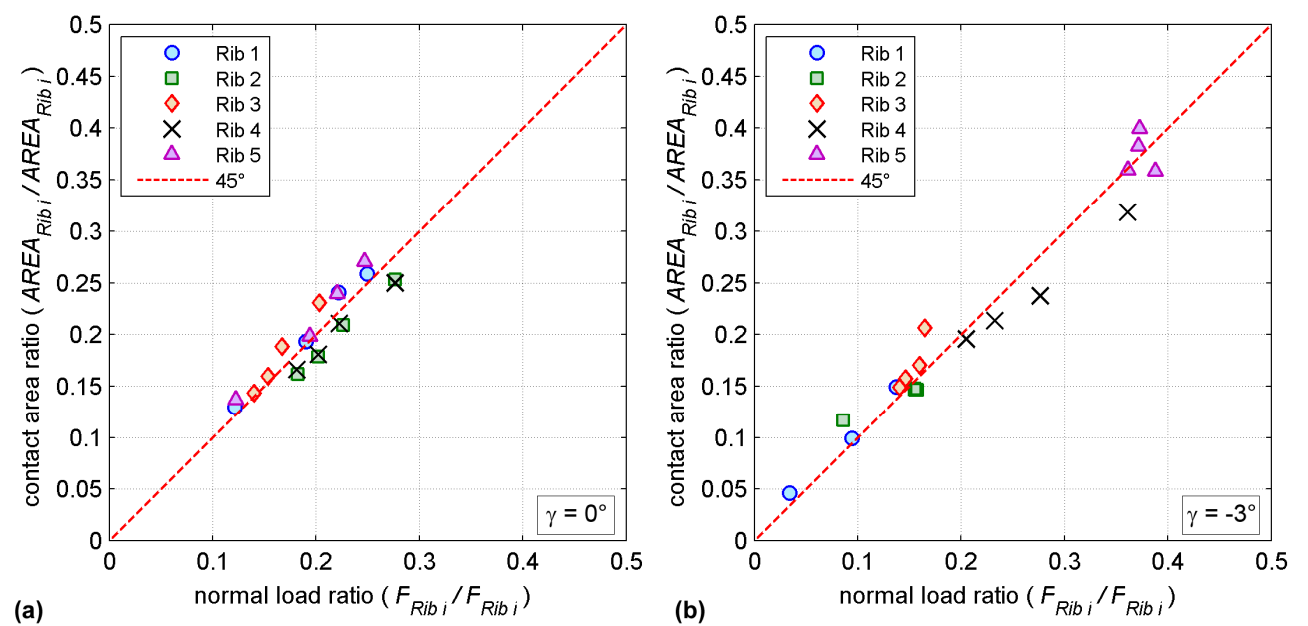

Figure 8: Contact area share of each tread rib against normal load share of each tread rib as a function of vertical tyre load: (a) tyre at zero camber and (b) tyre at $-3^{\circ}$ camber. 


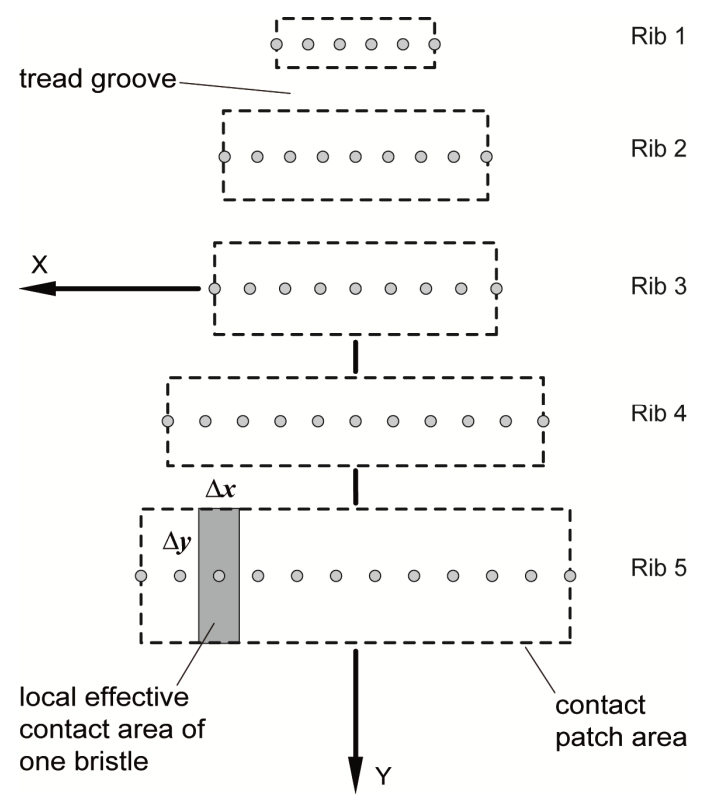

Figure 9: Top view of the idealised contact patch of a cambered tyre, showing a single bristle row per contact region. The dimensions of the effective contact area $(\Delta x$ and $\Delta y)$ of a single bristle are indicated for rib 5.

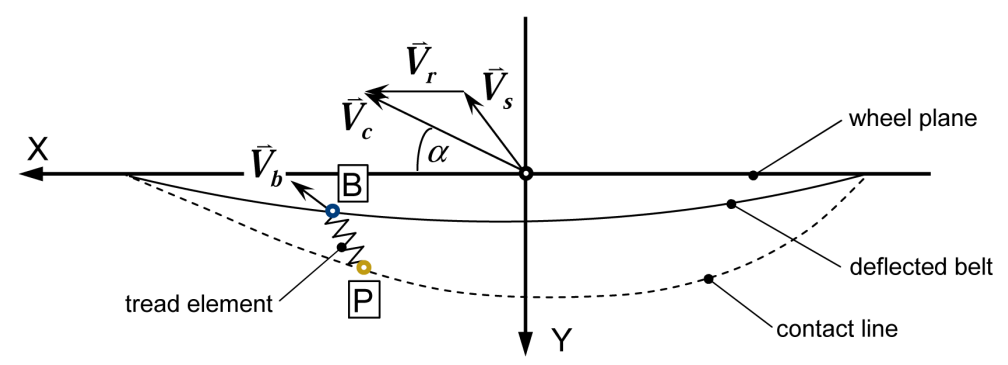

Figure 10: Schematic top view of the tread shear force model for a brake condition. For clarity, only one bristle row is shown and a slip angle $(\alpha)$ is introduced.

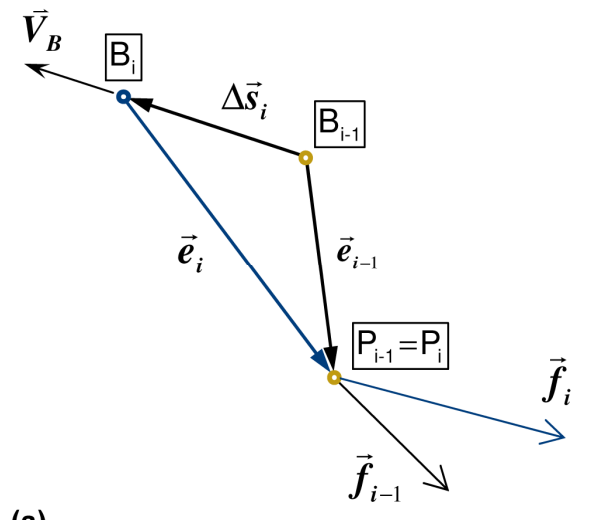

(a)

(b)

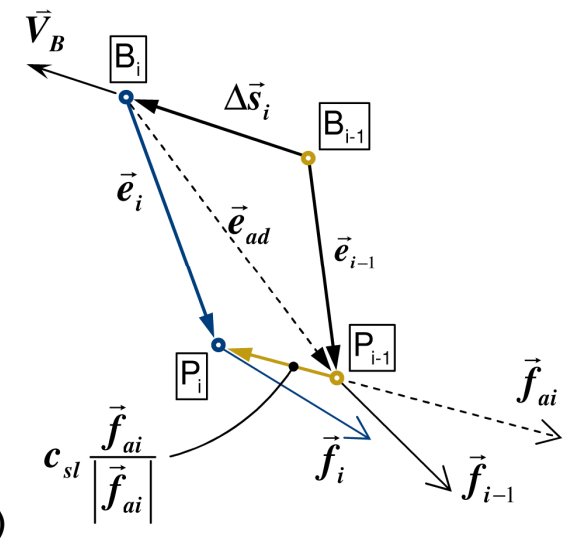

Figure 11: Two possible bristle tip states: (a) adhesion case and (b) sliding case. 


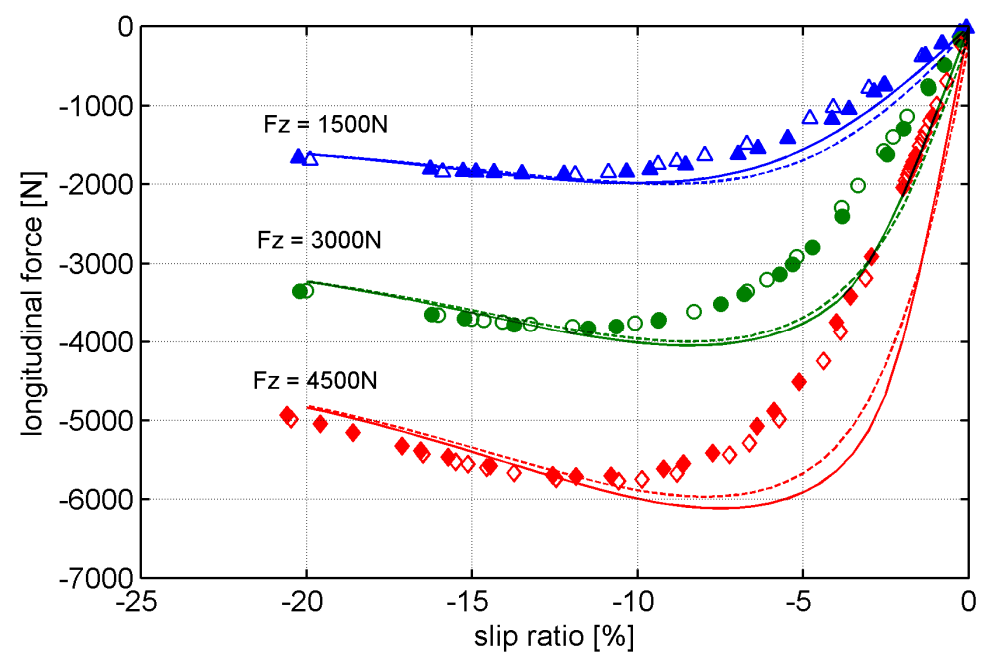

Figure 12: Longitudinal force against slip ratio at three different normal loads for an upright tyre and a tyre cambered at $-3^{\circ}$. FE model results are indicated by open markers (upright tyre) and solid markers (cambered tyre). Physical model results are denoted by solid lines (upright tyre) and dashed lines (cambered tyre).

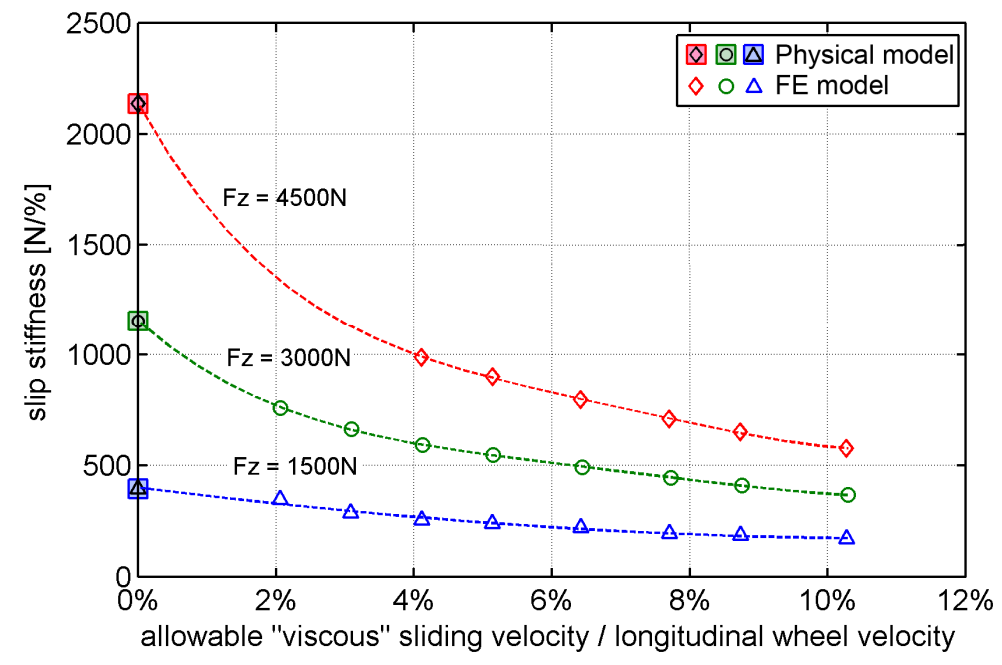

Figure 13: Slip tolerance setting against slip stiffness. 


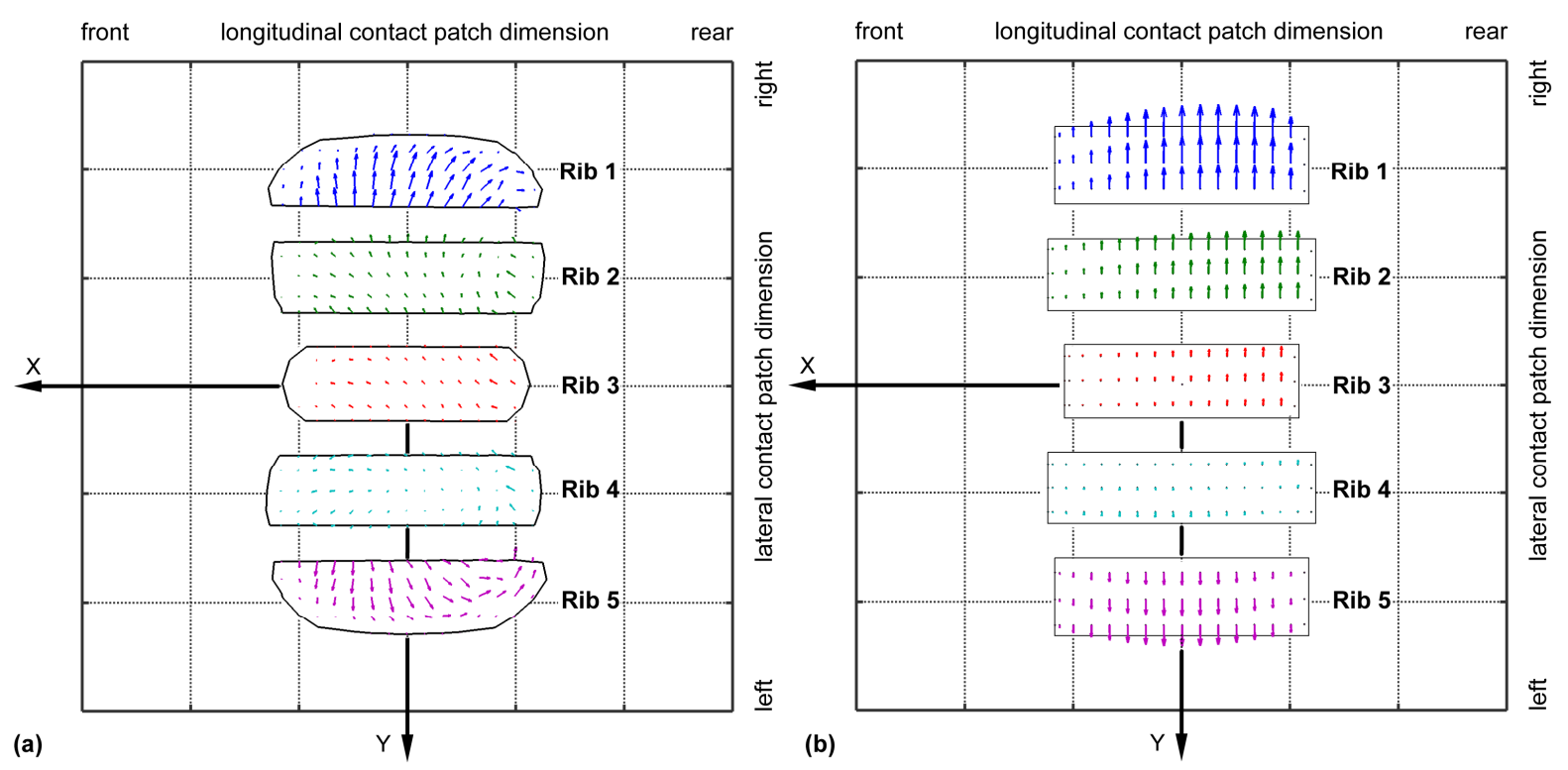

Figure 14: Shear stress distribution within the contact patch of a freely rolling, upright tyre at a normal load of $3000 \mathrm{~N}$ : (a) FE simulation and (b) developed physical model.
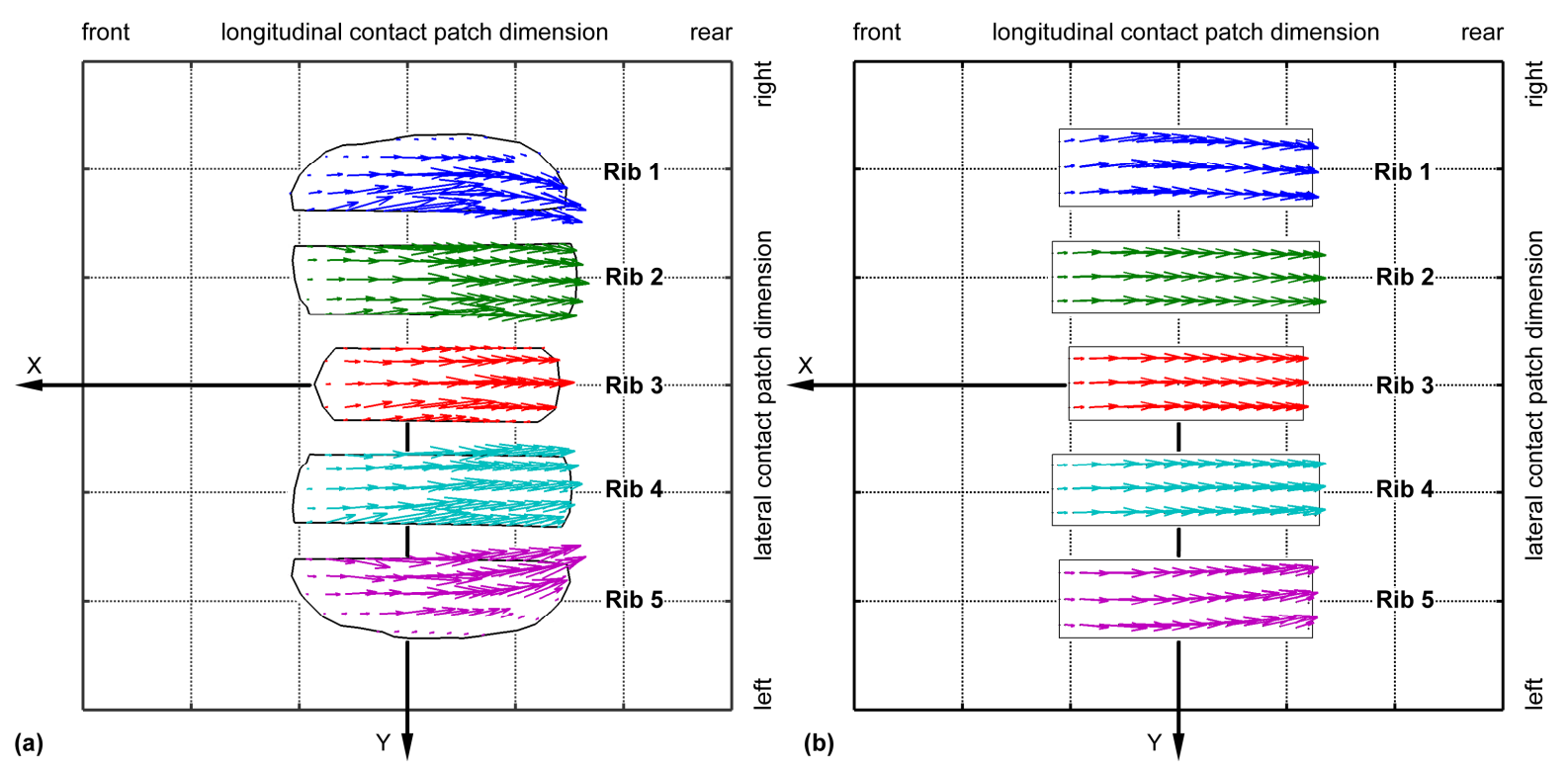

Figure 15: Shear stress distribution within the contact patch at a slip ratio of $-10 \%$ and a normal load of $3000 \mathrm{~N}$ : (a) FE simulation and (b) developed physical model. 


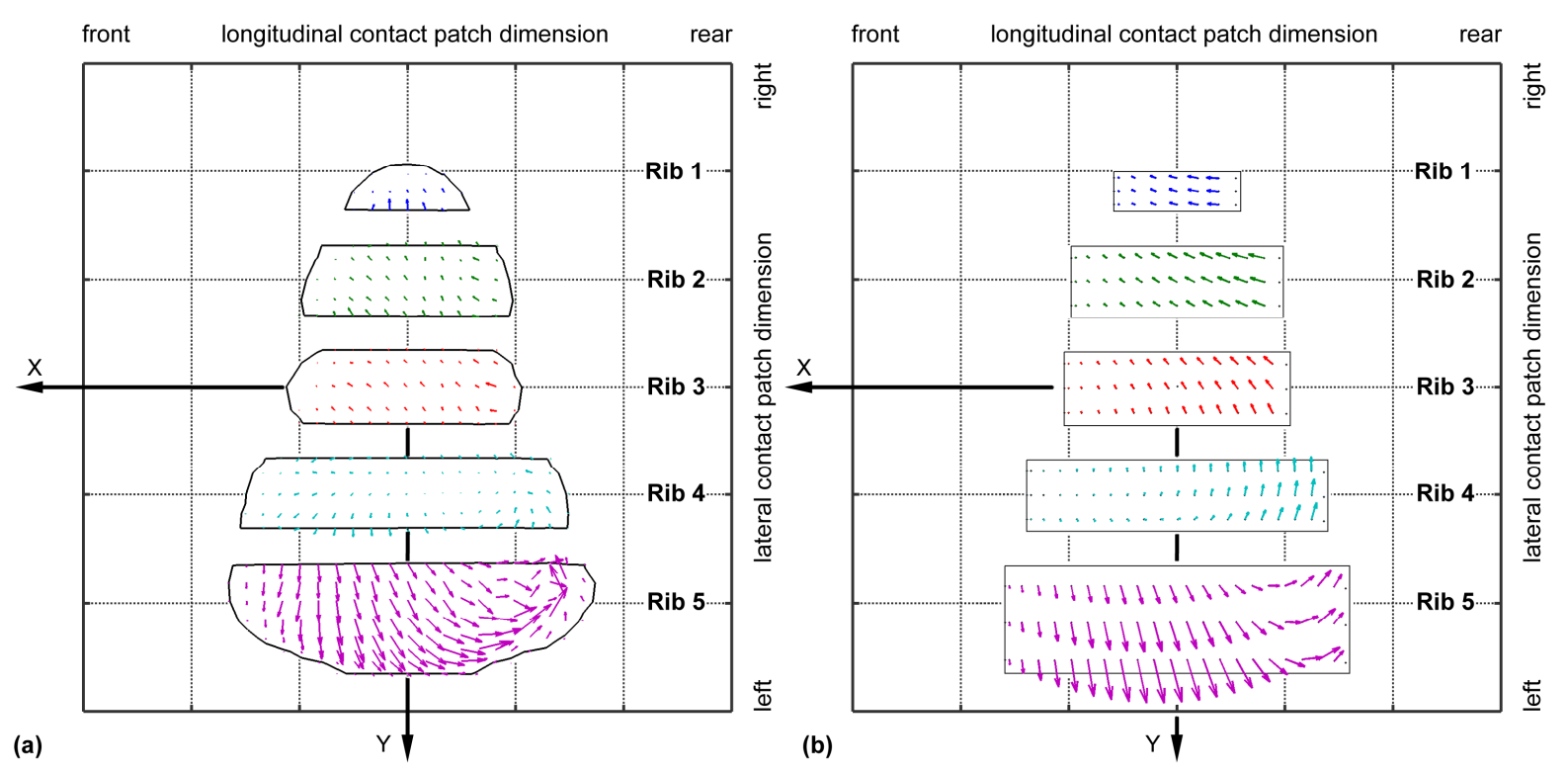

Figure 16: Shear stress distribution within the contact patch of a freely rolling tyre at $-3^{\circ}$ camber angle at a normal load of $3000 \mathrm{~N}$ : (a) FE simulation and (b) developed physical model.
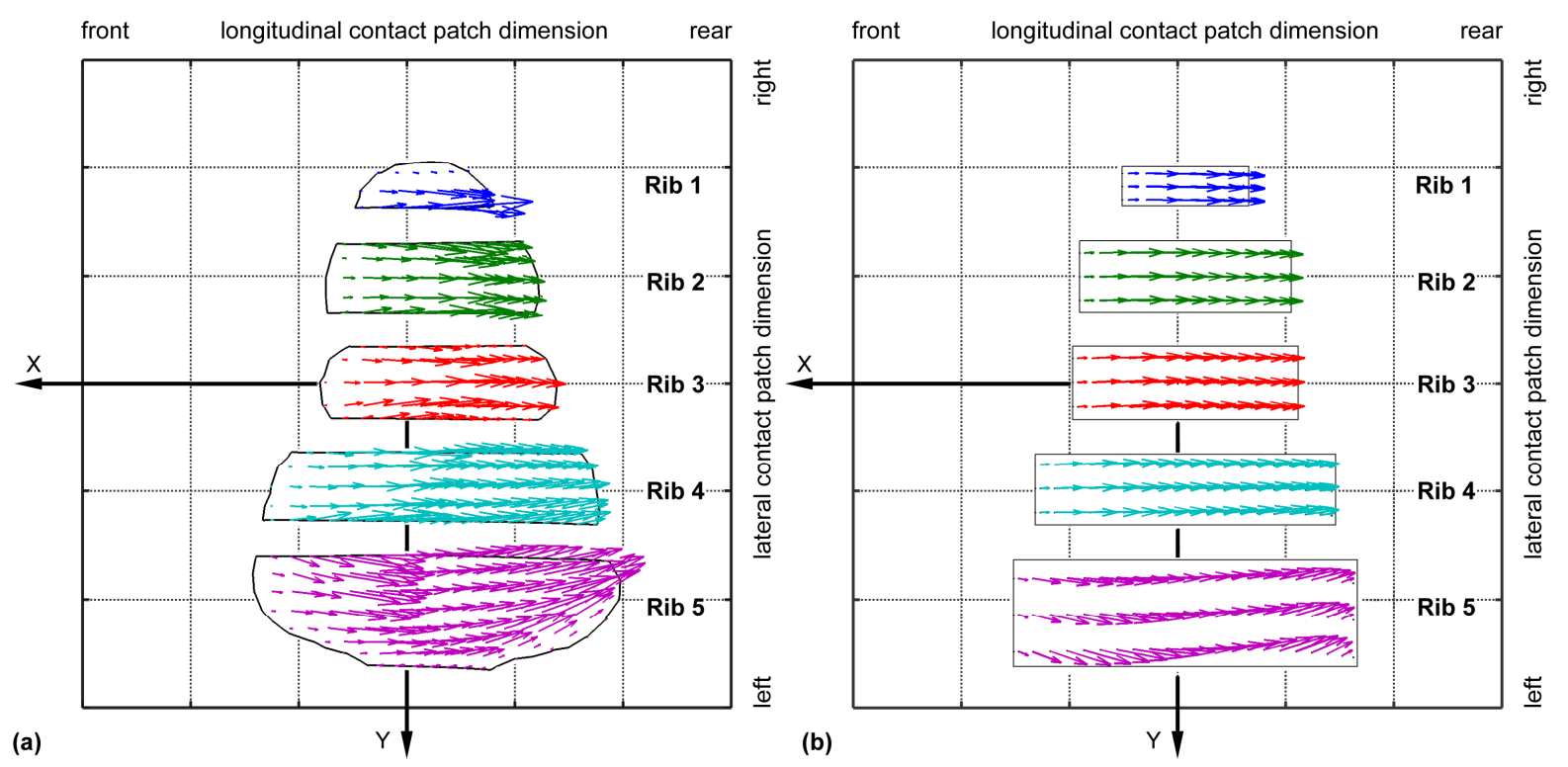

Figure 17: Shear stress distribution within the contact patch at $-3^{\circ}$ camber angle, a slip ratio of $-10 \%$ and a normal load of $3000 \mathrm{~N}$ : (a) FE simulation and (b) developed physical model. 